\title{
Discovery of the 3.9 days orbital periodicity in Very High Energy $\gamma$ rays from the X-ray binary LS 5039.
}

\author{
Mathieu de Naurois for the H.E.S.S. Collaboration* \\ LPNHE - CNRS/IN2P3 - Universités Paris VI/VII \\ E-mail: denauroidin2p3.fr
}

\begin{abstract}
Recent observations of the binary system LS 5039 with the High Energy Stereoscopic System (H.E.S.S.) revealed that its Very High Energy (VHE) $\gamma$-ray emission is modulated at the 3.9 days orbital period of the system. The bulk of the emission is largely confined to half of the orbit, peaking around the inferior conjunction epoch of the compact object. The flux modulation provides the first indication of $\gamma$-ray absorption by pair production on the intense stellar photon field. This implies that the production region size must be not significantly greater than the gamma-gamma photosphere size ( $\sim 1 \mathrm{AU})$, thus excluding the large scale collimated outflows or jets (extending out to $\sim 1000 \mathrm{AU}$ ). A hardening of the spectrum is also observed at the same epoch between 0.2 and a few $\mathrm{TeV}$ which is unexpected under a pure absorption scenario and could rather arise from variation with phase in the maximum electron energy and/or the dominant VHE $\gamma$-ray production mechanism. This first-time observation of modulated $\gamma$-ray emission allows precise tests of the acceleration and emission models in binary systems.
\end{abstract}

VI Microquasar Workshop: Microquasars and Beyond

September 18-22 2006

Società del Casino, Como, Italy

\footnotetext{
*Speaker.
} 


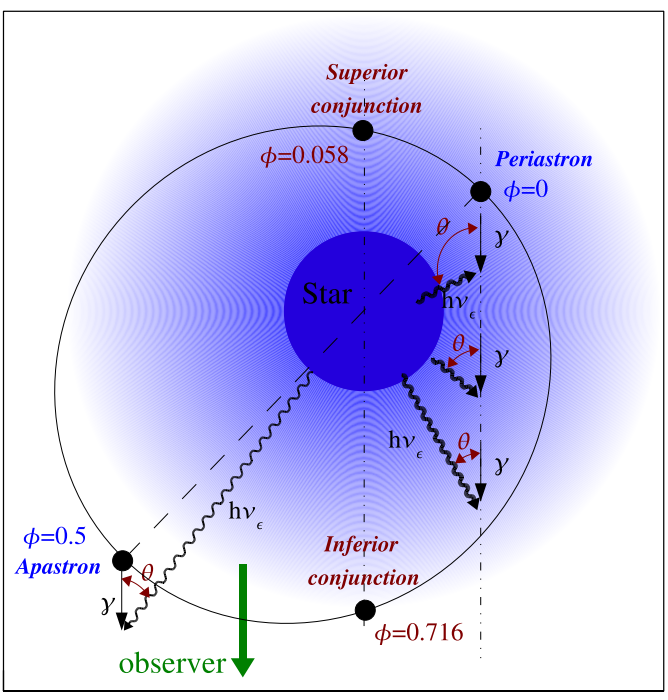

Figure 1: Orbital geometry of the binary system LS 5039 viewed from above and using the orbital parameters derived by Casares et al.[8]. Shown are: phases $(\phi)$ of minimum (periastron) and maximum (apastron) binary separation; epoch of superior and inferior conjunctions occurring when the compact object and the star are aligned along the observer light-of-sight.

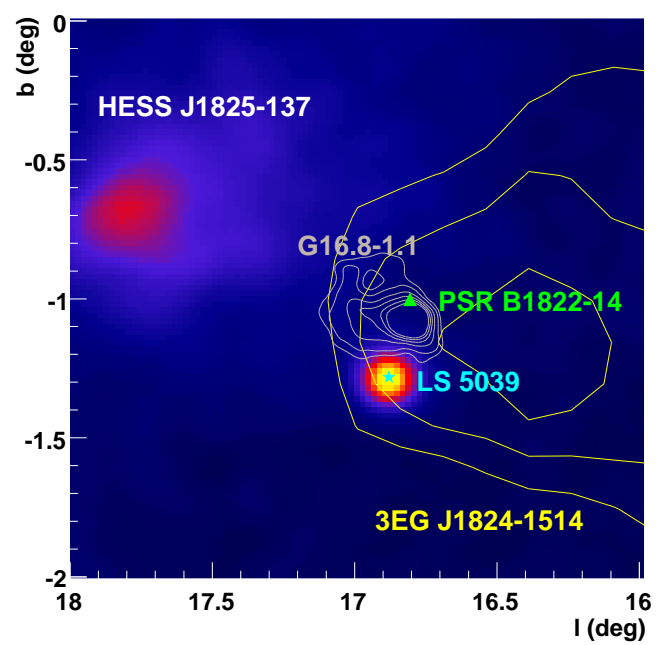

Figure 2: H.E.S.S. excess sky map around LS 5039, smoothed by the instrument point spread function. The blue star denotes the position of the VLBA source. The yellow contours correspond to the $68 \%, 95 \%$ and $99 \%$ confidence level region of the EGRET source 3EG J1824-1514. The extended source HESS J1825-137 observed in the same field of view can serve as a cross-check for timing-analysis.

\section{Introduction}

In the commonly accepted paradigm, microquasars consist of a stellar mass black hole fed by a massive star. They can exhibit superluminous radio jets[15], and hints for the presence of an accretion disk. LS 5039, identified in 1997[16] as a massive X-ray binary system with faint radio emission[14], was resolved by Paredes et al.[17] into bipolar mildly relativistic radio jets $(v \sim 0.2 c)$ emanating from a central core, thus placing it into the microquasar class. The detection of radio and variable X-ray emission[7] and its possible association with the EGRET source 3EG J18241514 suggested the presence of multi-GeV particles accelerated in jets. This binary system (Fig 1) consists of a massive $\mathrm{O} 6.5 \mathrm{~V}$ star in a $\sim 3.9$ day mildly eccentric orbit $(e=0.35)[8]$ around a compact object whose exact nature (black hole or neutron star) is still under debate.

\section{H.E.S.S. Observations}

The High Energy Stereoscopic System (H.E.S.S.) is an array of four identical Atmospheric Cherenkov Telescopes (ACT)[4] located in the Southern Hemisphere (Namibia, $1800 \mathrm{~m}$ a.s.1.) and sensitive to $\gamma$ rays above $100 \mathrm{GeV}$. LS 5039 was serendipitously detected in 2004 during the H.E.S.S. galactic scan[2] The 2004 observations have been followed up by a deeper observation campaign[3] in 2005, leading to a total dataset of 69.2 hours of observation after data quality selection. Data were analysed using two separate calibrations[1] and analysis pipelines. The results 
presented here are based on the log-likelihood comparison of the shower images with a precalculated semi-analytical model[9].

After selection cuts, a total of $1969 \gamma$-ray events were found within $0.1^{\circ}$ of the VLBA radio position of LS 5039, leading to a statistical significance of $40 \sigma$ (Fig. 2). The best fit position is, in Galactic Coordinates, $l=16.879^{\circ}, b=-1.285^{\circ}$ with statistical and systematic uncertainties of respectively $\pm 12^{\prime \prime}$ and $\pm 20^{\prime \prime}$. It is compatible with the VLBA position (denoted as a blue star in Fig. 2). We obtain an upper limit of $28^{\prime \prime}$ (at $1 \sigma$ ) on the VHE source extension.

\subsection{Timing Analysis}

The runwise VHE $\gamma$-ray flux at energies $\geq 1 \mathrm{TeV}$ was decomposed into its frequency components using the Lomb-Scargle periodogram [20] (Fig. 3) which is appropriate for unevenly sampled datasets such as those collected by H.E.S.S. A very significant peak (chance probability of $\sim 10^{-20}$ before trials) occurs in the Lomb-Scargle periodogram at the period 3.9078 \pm 0.0015 days, consistent with the most recent optical orbital period[8] (3.90603 \pm 0.00017$)$. The effect of subtracting a pure sinusoid at the orbital period is shown in Fig. 3, middle panel. The orbital peak disappears as expected, but also the numerous satellite peaks with chance probabilities less than $10^{-7}-10^{-8}$ that were present in the original periodogram. These peaks are beat periods of the orbital period with the various gaps present in the H.E.S.S. dataset (day-night cycle, moon period, annual period). The bottom panel of the same figure shows the result obtained on the neighbouring source HESS J1825137 observed in the same field of view as LS 5039, which doesn't show any statistically significant peak, thus demonstrating that the observed periodicity is genuinely associated with LS 5039 .

\subsection{Flux Modulation}

The runwise Phasogram (Fig 4) of integral flux at energies $\geq 1 \mathrm{TeV}$ vs. orbital phase $(\phi)$ shows an almost sinusoidal behaviour, with the bulk of the emission largely confined in a phase interval $\phi$ $\sim 0.45$ to 0.9 , covering about half of the orbital period. The emission maximum $(\phi \sim 0.7)$ appear to lag behind the apastron epoch and to align better with the inferior conjunction $(\phi=0.716)$, when the compact object lies in front of the massive star (see Fig. 1). The VHE flux minimum occurs at phase $(\phi \sim 0.2)$, slightly further along the orbit than superior conjunction $(\phi=0.058)$. Neither evidence for long-term secular variations in the VHE flux independent of the orbital modulation nor any other modulation period are found in the presented H.E.S.S. data.

\subsection{Spectral Modulation}

Due to changing environment with orbital phase (magnetic field strength, stellar photon field, relative position of compact object and star with respect to observer, ...), the VHE $\gamma$-ray emission spectrum is expected to vary along the orbit. We first define two broad phase interval: INFC centered on the inferior conjunction $(0.45<\phi \leq 0.9)$ and its complementary SUPC centered on the superior conjunction, corresponding respectively to high and low flux states. The high state VHE spectral energy distribution (Fig 5 ) is consistent with a hard power law with index $\Gamma=1.85 \pm$ $0.06_{\text {stat }} \pm 0.1_{\text {syst }}$ and exponential cutoff at $E_{0}=8.7 \pm 2.0 \mathrm{TeV}$. In contrast, the spectrum for low state is compatible with a relatively steep $\left(\Gamma=2.53 \pm 0.06_{\text {stat }} \pm 0.1_{\text {syst }}\right)$ pure power law extending from $200 \mathrm{GeV}$ to $\sim 20 \mathrm{TeV}$. Interestingly, the flux appears to be almost unmodulated at $200 \mathrm{GeV}$ as well as around $20 \mathrm{TeV}$, whereas the modulation is maximum around a few $(\sim 5) \mathrm{TeV}$. 


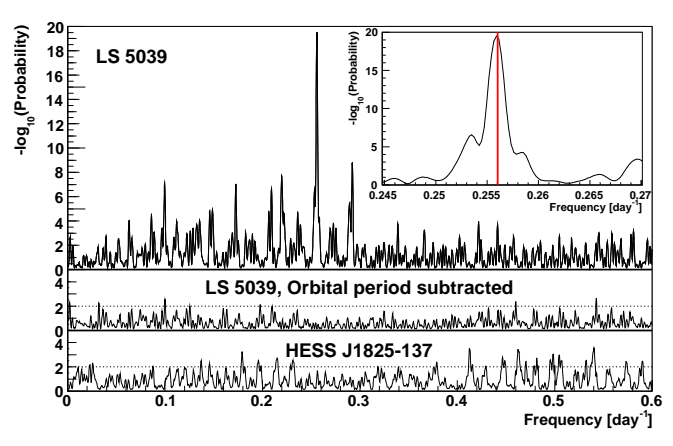

Figure 3: Lomb-Scargle (LS) periodogram of the VHE runwise flux of LS 5039 above $1 \mathrm{TeV}$ (Chance probability to obtain the LS power vs. frequency). From [3]. Zoom: inset around the highest peak, which corresponds to a period of $3.9078 \pm 0.0015$ days. This period is found to be compatible with the optical orbital period[8] denoted as a red line on the inset. Middle: LS periodogram of the same data after subtraction of a pure sinusoidal component at the orbital period of 3.90603 days (see text). Bottom: LS periodogram obtained on HESS J1825-137 observed in the same field of view.

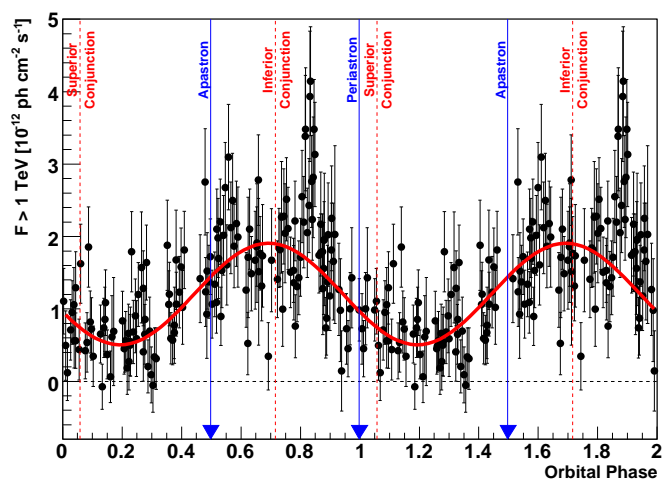

Figure 4: Phasogram (Integral run-by-run $\gamma$-ray flux above $1 \mathrm{TeV}$ as function of orbital phase) of LS 5039 from H.E.S.S. data from 2004 to 2005, using the orbital ephemeris[8]. Each run is $\sim 28$ minutes. Two full phase periods are shown for clarity. The vertical blue arrows depict the respective phases of minimum (periastron) and maximum (apastron) binary separation. The vertical dashed red lines show the respective phases of inferior and superior conjunction, when the star and the compact object are aligned along the observer's line of sight. From [3].

Trying to go to smaller phase interval, Fig 6 shows the results (photon index and differential flux at $1 \mathrm{TeV}$ ) of a pure power-law fit of the high energy spectra in 0.1 orbital phase bins (restricted to energies below $5 \mathrm{TeV}$ to avoid systematic effect introduced by the high state cutoff). The flux normalisation and photon index are strongly correlated, the flux being higher when the spectrum is harder and vice-versa. Interestingly, a similar effect, however in a smaller variation range and a different phasogram, was found in $\mathrm{X}$ rays[7].

\section{Interpretation and Conclusion}

The basic paradigm of VHE $\gamma$-ray production requires the presence of particles accelerated to multi-TeV energies and a target comprising photons (for $\gamma$-ray production through Inverse Compton effect) and/or matter of sufficient density (for $\gamma$-ray production through pion decay in hadronic processes). Several model classes are available to explain VHE emission from microquasars, differencing one from the other by the nature of accelerated particles and/or the location of the acceleration region. In jet-based models, particle acceleration could take place directly inside and along the jet, e.g. [6, and references therein], and also in the jet termination shock regions[12]. Non-jet scenarios are also available, e.g. [13, 11], where the emission arises from the interaction of a pulsar wind with the stellar companion equatorial wind.

New observations by HESS have established orbital modulation of the VHE $\gamma$-ray flux and energy spectrum from the XRB LS 5039. The observed VHE modulation indicates that the emission most probably takes place close (within $\sim 1 \mathrm{AU}$ ) to the massive stellar companion, where modu- 


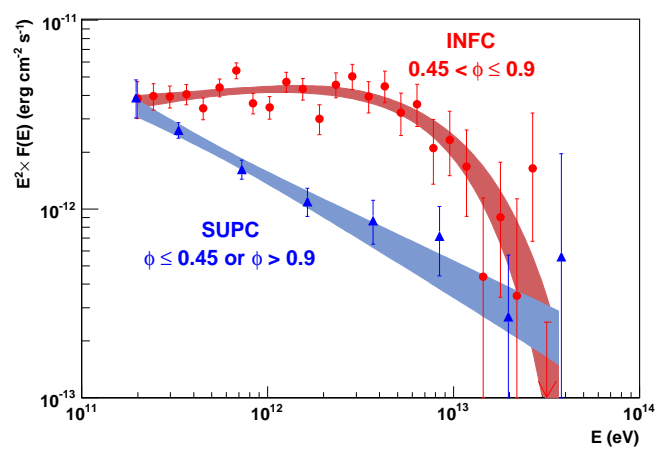

Figure 5: Very high energy spectral energy distribution of LS 5039 for the two broad orbital phase intervals defines in the text, INFC (red circles) and SUPC (blue triangles). The shades regions represent the $1 \sigma$ confidence bands on the fitted functions. A clear spectral hardening is occurring in the $200 \mathrm{GeV}$ to a few $\mathrm{TeV}$ range during the INFC phase interval. From [3].

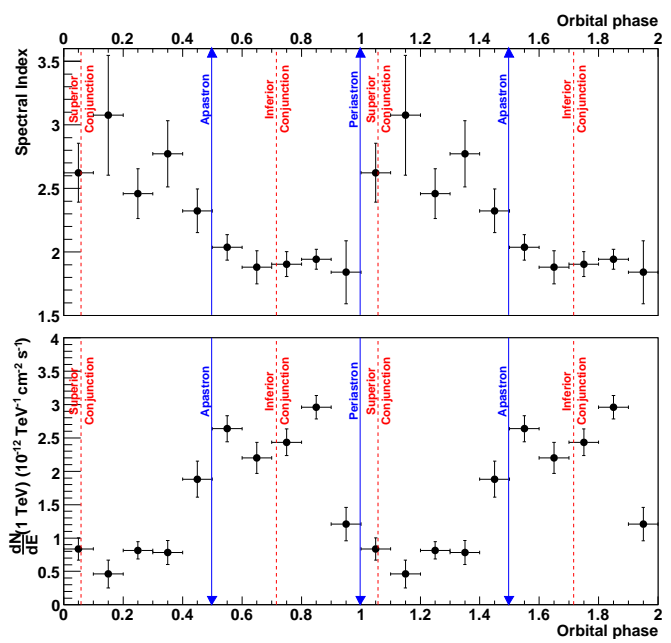

Figure 6: Top: Fitted pure power-law photon index vs. phase interval of width $\Delta \phi=0.1$. Bottom: Differential flux at $1 \mathrm{TeV}$ for the same phase interval. From [3].

lated $\gamma$-ray absorption via pair production $\left(e^{+} e^{-}\right)$on the intense stellar photon field is unavoidable (e.g. [11]) The observed spectral modulation is however incompatible with a pure absorption scenario, which in particular predicts a maximum variability around $300 \mathrm{GeV}$ and a VHE spectral hardening in the low flux state, inconsistent with observations.

Modulation could also arise from a modulation of the acceleration and cooling timescales along the orbit due to varying magnetic field and photon field densities (e.g. [3, and references therein]) which could modify the maximum electron energy and therefore induce a phase-dependent energy break in the $\gamma$-ray spectrum. Modulation of the accretion rate due to interaction of the stellar wind with the compact object in the microquasar scenario (e.g. [18]) could be another ingredient of the observed modulation.

A detailed study is now required to fully explain these new observations and understand the complex relationship between $\gamma$-ray absorption and production processes within these binary systems.

Acknowledgements The support of the Namibian authorities and of the University of Namibia in facilitating the construction and operation of H.E.S.S. is gratefully acknowledged, as is the support by the German Ministry for Education and Research (BMBF), the Max Planck Society, the French Ministry for Research, the CNRS-IN2P3 and the Astroparticle Interdisciplinary Programme of the CNRS, the U.K. Particle Physics and Astronomy Research Council (PPARC), the IPNP of the Charles University, the South African Department of Science and Technology and National Research Foundation, and by the University of Namibia. We appreciate the excellent work of the technical support staff in Berlin, Durham, Hamburg, Heidelberg, Palaiseau, Paris, Saclay, and in Namibia in the construction and operation of the equipment. 


\section{References}

[1] Aharonian, F., et al. (HESS Collaboration), Calibration of cameras of the H.E.S.S. detector, Astropart. Phys. 22 109-125 (2004)

[2] Aharonian, F., et al. (HESS Collaboration), Discovery of very high-energy gamma rays associated with an $X$-ray binary, Science 309, 746 (2005)

[3] Aharonian, F., et al. (HESS Collaboration), 3.9 day orbital modulation in the TeV gamma-ray flux and spectrum from the X-ray binary LS 5039, A\&A, in press (2006) and astro-ph / 0607192

[4] Aharonian, F., et al. (HESS Collaboration), Observations of the Crab Nebula with H.E.S.S., A\&A, in press (2006) and astro-ph/0607333

[5] Aharonian, F., et al. (HESS Collaboration), A possible association of the new VHE gamma-ray source HESS J1825-137 with the pulsar wind nebula G18.0-0.7, A\&A 442, L25-L27 (1005)

[6] Bosch-Ramon V., Paredes J.M.: A numerical model for the $\gamma$-ray emission of the microquasar LS 5039 Orbital X-Ray Variability of the Microquasar LS 5039. A\&A 417, 1075 (2004)

[7] Bosch-Ramon V., Paredes J.M., Ribó M., et al., Orbital X-Ray Variability of the Microquasar LS 5039, ApJ, 628, 388 (2005)

[8] Casares, J., Ribó, M., Ribas, I., Paredes, J.M., et al., A possible black hole in the $\gamma$-ray microquasar LS 5039, MNRAS 364, 899 (2005)

[9] de Naurois M., et al., Analysis methods for Atmospheric Cerenkov Telescopes, in proceedings of Towards a Network of Atmospheric Cherenkov Detectors VII (Palaiseau) p.149. (2005)

[10] Dubus, G.: Gamma-ray absorption in massive X-ray binaries. A\&A 451, 9-18 (2006)

[11] Dubus, G., Gamma-ray binaries: pulsars in disguise?, A\&A 456, 801 (2006) (2005)

[12] Heinz S., Sunyaev R.: Cosmic rays from microquasars : A narrow component to the CR spectrum? A\&A 390, 751-766 (2002)

[13] Maraschi L., Treves A.: A model for LSI 61³03. MNRAS 194, 1 (1981)

[14] Marti, J., Paredes, J. M., Ribó, M., The system LS 5039: a new massive radio emitting X-ray binary, A\&A 338, L71-L74 (1998)

[15] Mirabel, I.F., Rodriguez L.F., A Superluminal Source in the Galaxy, Nature 371, 46 (1994)

[16] Motch, C., et al., New massive X-ray binary candidates I from the ROSAT Galactic Plane Survey, $A \& A, \mathbf{3 2 3} 853-875$ (1997)

[17] Paredes, J.M., Martí J., Ribó M., Massi M., Discovery of a High-Energy Gamma-Ray-Emitting Persistent Microquasar, Science 288, 2340 (2000)

[18] Paredes, J.M., Bosch-Ramon V., Romero G.E., Spectral energy distribution of the gamma-ray microquasar LS 5039, A\&A 451, 259 (2006)

[19] Ribó, M., et al, LS 5039: A runaway microquasar ejected from the galactic plane, A\&A 384, p.954-964 (2002)

[20] Scargle, J.D., Studies in astronomical time series analysis. II - Statistical aspects of spectral analysis of unevenly spaced data, ApJ 263, 835 (1982) 